INPLASY

PROTOCOL

To cite: Zheng et al. Effects of Mind-body Exercise on Cognitive Function in the Elderly with Mild to Moderate Cognitive Impairment: A Systematic and Meta-Analysis. Inplasy protocol 202140108. doi:

10.37766/inplasy2021.4.0108

Received: 21 April 2021

Published: 21 April 2021

Corresponding author:

Xinhu Zheng

1821111059@sus.edu.cn

Author Affiliation:

Shanghai University of Sport

Support: 11DZ2261100.

Review Stage at time of this submission: Piloting of the study selection process.

Conflicts of interest:

None declared.

\section{Effects of Mind-body Exercise on Cognitive Function in the Elderly with Mild to Moderate Cognitive Impairment: A Systematic and Meta-Analysis}

Zheng, $X^{1}$; Wang, $X^{2}$.

Review question / Objective: 1. What troubles with the eldly people of cognitive impairment ? 2. What the effects of the mind-body exercise for the eldly people with cognitive impairment?

Condition being studied: At present, a large number of studies have confirmed that mind-body exercise has a positive effect on the cognitive function of the elderly with mild to moderate cognitive impairment, but the "dose relationship" of the impact of mind-body exercise on cognitive function is not yet clear, which is the focus of this study.

Information sources: The computer retrieves the CNKI, Wan Fang data, VIP, CBM, Pubmed, the Cochrane Library, Embase, Web of Science and EBSCO database, searching for randomized controlled trials on the intervention of mind-body exercise on the cognitive function of patients with cognitive impairment.

INPLASY registration number: This protocol was registered with the International Platform of Registered Systematic Review and Meta-Analysis Protocols (INPLASY) on 21 April 2021 and was last updated on 21 April 2021 (registration number INPLASY202140108).

\section{INTRODUCTION}

Review question / Objective: 1. What troubles with the eldly people of cognitive impairment ? 2. What the effects of the mind-body exercise for the eldly people with cognitive impairment?

Condition being studied: At present, a large number of studies have confirmed that mind-body exercise has a positive effect on 
the cognitive function of the elderly with mild to moderate cognitive impairment, but the "dose relationship" of the impact of mind-body exercise on cognitive function is not yet clear, which is the focus of this study.

\section{METHODS}

Participant or population: In this study, we selected the older adults with mild-tomoderate cognitive impairment.

Intervention: In the study, we sclect the articles with the mind-body exercise intervention by Tai Ji, Tai Chi, TaiJiQuan, Wuqinxi, Qigong and so on.

\section{Comparator: None.}

\section{Study designs to be included: RCT.}

Eligibility criteria: The literature inclusion criteria: (1) The subjects were elderly with mild to moderate cognitive impairment, or those whose cognitive function assessment satisfied the Mini-mental State Examination (MMSE) score of 10-27, or with the Montreal Cognitive Assessment Scale (MOCA) <26 points, conforming to the evaluation criteria of the fourth edition of the Diagnostic and Statistical Manual of Mental Disorders (DSM-IV-R) revised by the American Diagnostic and Statistical Manual of Mental Disorders, with normal activities of daily living. (2) A study was regarded as two studies if there were two experimental groups. (3) The type of study was randomized controlled trial (RCT). The literature exclusion criteria: (1) The subjects of the experimental group were patients with severe cognitive impairment or normal elderly with Mini-mental State Examination (MMSE) 27 points, and Montreal Cognitive Assessment Scale (MOCA) $\geq 26$ points. (2) There was no single exercise intervention group. (3) Repeatedly published and poorly assessed literature. (4) Literature with unclear experimental data which could not be calculated.

Information sources: The computer retrieves the CNKI, Wan Fang data, VIP,
CBM, Pubmed, the Cochrane Library, Embase, Web of Science and EBSCO database, searching for randomized controlled trials on the intervention of mind-body exercise on the cognitive function of patients with cognitive impairment.

Main outcome(s): Various scales and tests used to assess cognitive function include Mini-Mental State Examination (MMSE), Montreal Cognitive Assessment (MOCA), Alzheimer's Disease Assessment ScaleCognitive Section (ADAS-cog), Wechsler Memory Scale, Trail Making Test (TMT), Auditory Verbal Learning Test (AVLT), Stroop word color test, animal naming test, etc.

Quality assessment / Risk of bias analysis: Two researchers independently screened the literature based on the inclusion and exclusion criteria of the literature, crosschecked, and extracted relevant data. If there were differences in the stages of mutual review, screening of literature, and data extraction, the third researcher would discuss whether to include.

Strategy of data synthesis: Use Review Manager 5.3 to process the data in the literature, perform effect size consolidation and heterogeneity test on the outcome indicators, and draw a forest map. The literature outcome indicators included in this study were all continuous variables. The effect size was expressed by Mean Difference (MD) and Standardized Mean Difference (SMD), and the $95 \%$ confidence interval $(95 \% \mathrm{Cl})$ was calculated at the same time. This meta-analysis strictly followed the PRISMA guidelines. The level of the meta-analysis was set as $a=0.05$, and the $P$ value and 12 were used for the heterogeneity test. If there was no statistical heterogeneity among the results of the studies $(12 \leq 50 \%, P>0.10)$, fixed effects model was selected. If there was statistical heterogeneity among the studies, the source of the heterogeneity shall be further analyzed, and after the influence of obvious clinical heterogeneity has been excluded, the random effects model shall be used for analysis. 
Subgroup analysis: Planede subgroup analyses will be: (1) Type of exercise intervention; (2) Type of exercise frequency; (3) Type of exercise intensity; (4) Type of exercise duration.

Sensitivity analysis: The review manager was used for sensitivity analysis of results with high heterogeneity.

Country(ies) involved: China.

Keywords: mind-body exercise; cognitive impairment; cognitive function; metaanalysis.

Contributions of each author:

Author 1 - Xinhu Zheng.

Email: 1821111059@sus.edu.cn

Author 2 - Xing Wang.

Email: 18930132117@163.com 